\title{
XXXIII. An improved method of determining moisture and carbonic acid in air
}

\section{J. S. Haldane M.A. M.D. \& M. S. Pembrey B.A.}

To cite this article: J. S. Haldane M.A. M.D. \& M. S. Pembrey B.A. (1890) XXXIII. An improved method of determining moisture and carbonic acid in air , Philosophical Magazine Series 5, 29:179, 306-331, DOI: 10.1080/14786449008619944

To link to this article: http://dx.doi.org/10.1080/14786449008619944

曲 Published online: 08 May 2009.

Submit your article to this journal $[\pi$

Џll Article views: 2

Q View related articles $\square$ 


\section{Messrs. Haldane and Pembrey on an Improved Method}

though quite unmagnetic in large lumps, out of the crushed rocks with magnets. Magnetic ore-separators are also said to be used on a practical seale.

In conclusion I may mention that M. Heusler, of the Isabellenhütte, Dillenburg, kindly sent me an alloy which he defined as follows:-93-93.5 per cent. $M n, 1-1 \cdot 2$ per cent. $\mathrm{Fe}, \cdot 2-\cdot 5$ per cent. $\mathrm{Si}$, and earbon. This gave not the slightest trace of an attraction by the pole of a steel magnet or of the Ruhmkorff, whether in a piece or pounded almost to dust.

I beg to tender my best thanks to Prof. F. Kohlrausch, in whose laboratory these experiments were carried out.

Phys. Inst. of Strasburg Univ., Nov. 1, 1889.

XXXIII. An Improved Method of Determining Moisture and Carbonic Acid in Air. By J. S. Haldane, M.A., M.D., and M. S. Pembrex, B.A., Fell Exhibitioner of Christ Church, Oxford. (From the Physiological Laboratory, Oxford.) *

WE were originally led to undertake the present investigation by difficulties experienced in measuring the respiratory exchange of oxygen and carbonic acid in animals. In the method which we wished to employ the results depend on an accurate determination of the difference in the percentage of moisture and carbonic acid in the air entering and that leaving a ventilated chamber in which the animal is placed. As the ventilation current is large, the difference to be measured is a small one, and errors of analysis are correspondingly important, especially in short experiments.

Although the object of our work was thus originally physiological, we have been guided ehiefly by regard to the wider applications, particularly in meteorology, of the methods in question.

\section{The Determination of Moisture.}

Of the various methods in use for determining moisture in air the " chemical" method is generally acknowledged to be the most accurate when properly carried out. This method consists in the aspiration of a known volume of the air through one or more weighed tubes filled with a substance which absorbs moisture, such as anhydrous phosphoric acid or pumice soaked in sulphuric acid. The increase in weight of the absorption-tubes gives the weight of moisture contained in the air. The great disadvantage of this method as used

* Communicated by Prof. Odling, F.R.S. 
of Determining Moisture and Carbonic Acid in Air. 307

hitherto lies in the fact that for an accurate analysis a very long period of experiment is necessary. The changes in the amount of moisture which in ordinary cases occur during the period of experiment are thus not indicated, and the method is rendered useless for many purposes, besiles being very inconvenient.

The details of the method have recently been reinvestigated by Shaw, in a research undertaken at the request of the Meteorological Council * . He found that with a U-tulbe filled with pumice and sulphuric acid and weighing about 170 grammes, the absorption was complete with a rate of aspiration of about 8 or 10 litres per hour $t$. With double this rate there was a considerable escape of moisture through the first absorption-tube $\ddagger$. Thus, with the faster rate of aspiration, the increase of weight of a second absorption-tube had to be added to the result. But since it was found that between the weighings the second tube frequently varied considerably in weight from accidental causes, the advantages of the faster rate were neutralized.

We have endeavoured to improve the method in three directions :-(1) by reducing to a minimum accidental variations in the weight of the tubes; (2) by increasing the efficiency of the absorbing tubes, and thus making a very rapid rate of aspiration possible; and (3) by making the apparatus more convenient.

Fig. 1.

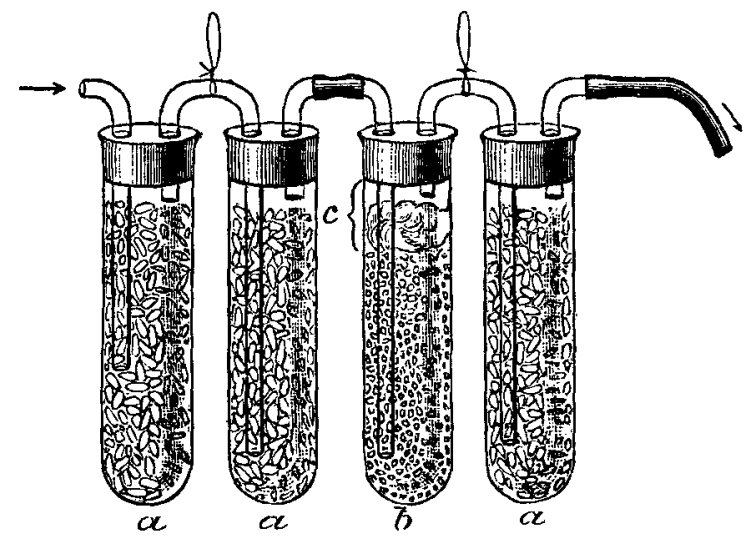

Absorption apparatus for Moisture and Carbonic Acid, $\times \frac{1}{2}$.

$a$. Tubes filled with pumice soaked in sulphuric acid. $b$. Tube filled with soda-lime. $c$. Plug of cotton wool.

The absorption apparatus which we finally adopted is shown in fig. 1. We shall describe it in detail because, as will be

* Phil. Trans. vol. A, 1888, p. $73 . \quad \dagger$ Ibid. . $84 . \quad \ddagger$ Ibid. p. 88. 


\section{Messrs. Haldane and Pembrey on an Improved Method}

seen below, the disadvantages of the chemical method, as ordinarily used, are dependent on a want of attention to details. It consists of a pair of test-tubes, containing pumice soaked in sulphuric acid. The test-tubes are $4 \times 1$ inch, and made of thin glass. Each tube is provided with a double-bored cork about $\frac{1}{2}$ inch thick, which is fitted with glass tubing of about $\frac{3}{16}$ inch internal diameter, and in the form shown in the figure* ${ }^{*}$ The tubing must fit firmly. The corks are covered with a layer of hard paraffin inside and out. They are pushed down a very little below the tops of the tubes, the end of each tube being first wiped inside free of acid, and then warmed to soften the paraffin on the cork and so facilitate its entrance. To enable the longer limb of the tubing to be pushed down, a passage should first be cleared in the pumice with a piece of glass rod. A layer of paraffin is spread smoothly over the top of the cork until it is just level with the edges of the tube. The apparatus must be absolutely tight, and should allow air to pass perfectly freely when suction is applied. It should finally be carefully cleaned with a wet cloth to remove any traces of acid, and then dried.

The pumice is sifted through a wire sieve with about 7 meshes to the inch, and shaken in a fine one to remove the powder. It is then heated to redness by playing on it with a large blowpipe-flame, and thrown still hot into "pure redistilled" sulphuric acid. The superfluous acid is then poured off, and the pumice preserved for use in a stoppered bottle. Before using the tubes for experiments we have always taken the precaution of washing them out with air, as the air passed through them at first usually tastes slightly of sulphurous acid.

Each pair of tubes when filled weighs about 80 grammes, and can therefore be weighed on any ordinary balance.

To diminish to a minimum errors arising from accidental variations in weight of the tubes, we adopted the plan of weighing against a counterpoise consisting of a similar absorption-tube, and of about the same weight. This counterpoise is always kept in the same place as the absorptiontubes. Since during an experiment the absorption-tubes will

* The longer piece of glass tubing in the first sulphuric acid absorptiontube is made somewhat shorter than the corresponding tubing in the other absorption-tube. The inconvenience arising from the collection of water in the first tube is thus avoided.

Since writing the above we have been supplied by Messrs. Gallenkamp with pairs of tubes made in one piece. These are more brittle, but otherwise very convenient. 
of Determining Moisture and Carbonic Acid in Air. 309

have been warmed above the temperature of the counterpoise, the weighing must be deferred for half an hour, so as to allow their temperatures to become equal again. The tubes are weighed unstoppered. If several tubes are to be weighed the stoppers* are removed before weighing from the whole of the tubes, including the counterpoise, and not replaced until the last tube has been weighed. No absorbent is kept inside the balance-case. The tubes should be lifted by the wires attached to them for hanging on the balance.

We found the following form of aspirator very convenient, especially when the apparatus had to be carried to a distance. Two bottles (fig. 2), each holding about 3200 cub. cent. up to a mark in the neck, were arranged as shown in the figure, and connected together by a piece of stout, non-collapsing rubber-

Fig. 2.

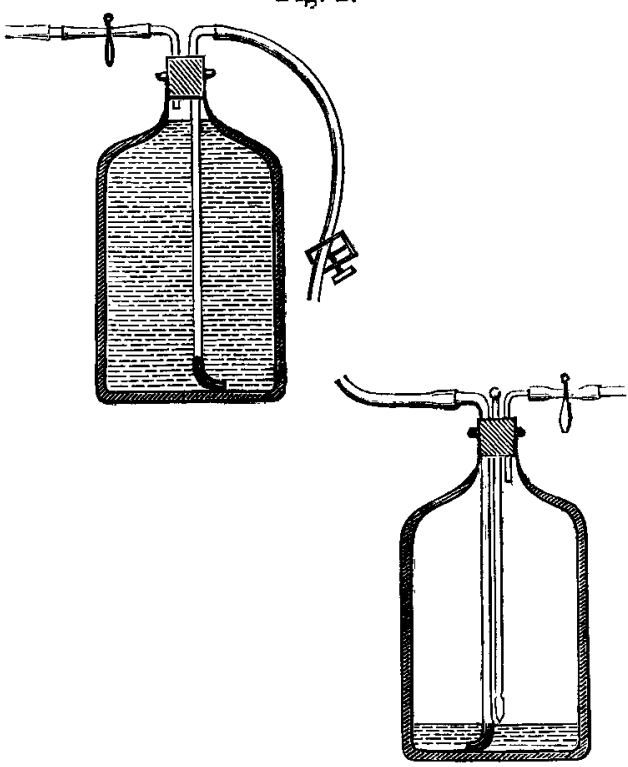

tubing provided with a screw-clip for regulating the rate of aspiration. The burette clips are for starting or stopping the flow. The bottles are covered outside with a layer of felt, to keep the temperature of the air and water equal during an experiment, and also to prevent breakage. A vertical strip on each bottle is, however, left uncovered, so that the height of the water may be watched during the experiment and the

* The stoppers used are the ordinary ones, consisting of a short piece of black rubber about $\frac{3}{4}$ inch long, closed at one end by a piece of glass rod.

Phil. Mag. S. 5. Vol. 29. No. 179. April 1890. 2 B 


\section{Messrs. Haldane and Pembrey on an Improved Method}

reading of the thermometer taken. It is convenient to graduate the bottles roughly by pasting strips of paper at intervals of half a litre up the uncovered strip of glass. With the help of this graduation it is easy to ascertain if the aspirator is running at about the proper rate.

To graduate the aspirator, one of the boitles is allowed to drain for a minute, and is then weighed accurately. The two bottles are now connected and the weighed bottle is filled with distilled water to the mark, the tubing also being filled with water. The cork of the weighed bottle is then removed, care being taken to let no water escape from the end of the long piece of glass tubing. The bottle filled with water is then weighed again; and by deducting this weight from that of the empty bottle and allowing for air displaced \&c., as in the graduation of a measuring-flask, the amount of water held by the bottle filled to the mark wi the tubing in it, is obtained. The difference between this amount and some round number of cub. cent., such as 3000 , is then measured into the bottle, wh:ch has been previously emptied and drained for a minute. The cork is tien replaced, care being taken, as before, not to spill any of the water in the tubing. The aspirator is now ready for use, and measures off with the utmosi exactness 3000 cub. cent. of air each time it is reversed.

We are now in a position to consider the advantages of the method as thus modified. As regards, firstly, errors of weighing, we found, in accordance with previous observers, that an absorption-tube, when left to itself, varies considerably in weight from hour to hour. The same is true of a tube through which dry air from another absorption-tube is passed * There is usually a gain in weight, but sometimes a loss. This may be due partly to variations in temperature and barometric pressure. It is also due to gradual penetration of moisture into the tubes and to the varying amount of moisture deposited on the surface of the glass. The amount of this variation in weight was often two milligrammes or more, both in Shaw's experiments and our earlier ones with other absorption-tubes. With the method just described the tubes did not vary in apparent weight by more than three decimilligrammes in a day ; and these slight variations were in part accounted for by small imperfections in our set of weights.

The advantages of using a counterpoise are illustrated by an experiment in which weighings with the counterpoise were compared at intervals with ordinary weighings during

* Cf. the data on this point given by Shaw, loc. cit. p. 84 . 
of Determining Moisture and Carbonic Acid in Air. 311 several days. The differences found are shown in the following Table:-

\begin{tabular}{|c|c|c|c|c|c|}
\hline \multicolumn{2}{|c|}{ Intervals between woighings. } & 20 hours. & 3 hours. & $4 \frac{1}{2}$ hours. & 42 hours. \\
\hline \multirow{2}{*}{\multicolumn{2}{|c|}{ 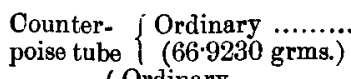 }} & +0.0012 & +0.0010 & +0.0008 & +0.0024 \\
\hline & & & +0.0009 & +0.0008 & +0.0018 \\
\hline \multirow[t]{2}{*}{ Tube 1} & $\begin{array}{c}(77 \cdot 7075) \\
\text { Comparative.......... } \\
\left(10^{\circ} 7840\right)\end{array}$ & ...... & \pm 00000 & \pm 0.0000 & -0.0004 \\
\hline & $\begin{array}{l}\text { Ordinary } \\
\quad(87.6274)\end{array}$ & +0.0007 & +0.0015 & & \\
\hline Tube 2 & $\begin{array}{c}\text { Comparative....... } \\
(20.7041)\end{array}$ & -0.0001 & +0.0002 & ...... & +0.0002 \\
\hline \multirow{2}{*}{ Tube 3} & $\begin{array}{c}\text { Ordinary } \ldots \ldots \\
(69 \cdot 6367)\end{array}$ & & & +0.0006 & \\
\hline & $\begin{array}{l}\text { Comparative..... } \\
(2 \cdot 7136)\end{array}$ & \pm 0.0000 & -0.0002 & $\pm 0 \cdot 0000$ & $-0 \cdot 0005$ \\
\hline
\end{tabular}

With regard, secondly, to the rate of aspiration possible and the lasting powers of the tubes, we made a number of experiments.

When a single absorption-tube was used, instead of the double ones described above, the absorption in the first tube was perfect (at least with rates up to two litres per minute) for a considerable time, as shown by the following experiments.

$$
\text { Experiment No. } 1 .
$$

90 litres of air were aspirated at a rate of 1 litre per minute through two single absorption-tubes connected together by a very short piece of rubber tubing. The aspirator in this and the following experiment was a glass filter-pump, the air being measured by a gas meter :-

$$
\begin{aligned}
& \text { Tube 1 . . . +0.5142. } \\
& \text { Tube 2. . . }+0 \cdot 0000 \text {. }
\end{aligned}
$$

Experinent No. 2.

90 litres aspirated at 1 litre per minute.

$$
\begin{aligned}
& \text { Tube } 1 \text {. . . +0.4712. } \\
& \text { Tube } 2 . \quad . \quad+0.0002 \text {. }
\end{aligned}
$$

$$
\text { Experiment No. } 3 .
$$

50 litres aspirated at $1 \frac{3}{4}$ litres per minute.

$$
\begin{aligned}
& \text { Tube 1 . . . + +0.4057. } \\
& \text { Tube 2. . . + +0.0001. } \\
& 2 \mathrm{~B} 2
\end{aligned}
$$


312 Messrs. Haldane and Pembrey on an Improved Method

Experiment No. 4.

21 litres aspirated at 2 litres per minute.

$$
\begin{aligned}
& \text { Tube } 1 \text {. } \\
& \text { Tube 2 }
\end{aligned}
$$

Experiment No. 5.

50 litres aspirated at 1 litre per minute.

$$
\begin{aligned}
& \text { Tube 1 . . . + +0.4163. } \\
& \text { Tube } 2 \text {. . . }-0.0003 \text {. }
\end{aligned}
$$

The same tubes were not used through all these experiments, as tube No. 1 had become spent in the middle of the series. The use of double tubes enormously increases the lasting powers, so that, as shown by the following experiment, a double tube may be used for 300 determinations or more without being recharged.

\section{Experiment No. 6.}

The number of analyses possible without refilling a double absorption-tube was determined by aspirating air through two of them at a rate of 1 litre a minute, and weighing the second pair at intervals. 120 litres had previously passed through pair No. 1.

$\begin{array}{cc}\text { Litres of Air } & \text { Variation in W } \\ \text { Aspirated through Pair 1. } & \text { of Pair 2. } \\ 235 & -0.0005^{*} \\ 354 & -0.0002 \\ 418 & +0.0000 \\ 568 & -0.0001 \\ 753 & -0.0003 \\ 1046 & +0.0000 \\ 1225 & \\ 1346 & +0.0001 \\ 1588 & +0.0003 \\ 1767 & -0.0002 \\ 1994 & +0.0000 \\ 2200 & -0.0001\end{array}$

At the end of the experiment the first pair had absorbed 12.8255 grammes from 2320 litres of air. Although this pair was still absorbing moisture completely we did not continue the experiment further, on account of the inconvenient accumulation of dilute acid in the first tube.

As in determining moisture we have generally made a carbonic-acid determination simultaneously, our usual rate of

* Weighed by mistake five minutes after experiment. 
of Determining Moisture and Carbonic Acid in Air. 313 aspiration has (for reasons given, p. 321) been about 1 litre per minute. The following were test experiments with faster rates :-

Experiment No. 7.

25 litres aspirated at 3 litres per minute.

$$
\begin{aligned}
& \text { Pair (1) . . . . +0.2012. } \\
& \text { Pair (2) . . . . +0.0004. }
\end{aligned}
$$

Experiment No. 8.

25 litres aspirated at 4 litres per minute.

$$
\begin{aligned}
& \text { Pair (1) . . . . +0.3453. } \\
& \text { Pair (2) . . . +0.0003. }
\end{aligned}
$$

Experiment No. 9.

21 litres aspirated at 7 litres per minute.

$$
\begin{array}{llll}
\text { Pair (1) . . } & . & . & +0.2142 . \\
\text { Pair (2) . } & \text {. } & \text {. } & +0.0001 .
\end{array}
$$

The rate of aspiration may thus be, if required, 7 litres per minute.

With the view of determining whether there is any appreciable constant error due to incomplete absorption of moisture by the first tube, we have added up the variations in weight of the second tube in all our experiments in which a second absorption-tube was used.

Total moisture absorbed by first tube.

13.0522 grms.

Sum of variations of second tube.

-0.0007 grms.

The mean variation in weight of the second tube was thus only 0.0065 per cent. ; and a constant error of this order would be quite inappreciable in ordinary experiments.

Additional evidence of the accuracy of the method is afforded by the fact that in the simultaneous determinations recorded below (pp. 322 and 327 ) the two sets of results are practically identical. We have also made for another purpose two experiments in which air dried by sulphuric acid was passed through a weighed vessel containing water, and then again through a drying apparatus. The apparatus used was on a much larger scale, and the rate of aspiration was in one case 7 and in the other 15 litres per minute. The balance employed weighed to centigrammes. The vessel containing water lost 4.86 and 3.62 grammes, and the drying vessels gained $4 \cdot 86$ and $3 \cdot 61$ grammes respectively.

If the modified method described above be compared with the old method as investigated by Shaw, it is seen that by the 


\section{Messrs. Haldane and Pembrey on an Improved Method}

modified method the rate of aspiration may be more than 20 times as fast and the accidental errors in weighing are reduced to a sixth or less. Thus for a given duration of experiment the error in the modified method is less than a hundredth of that by the older method. In other words, an experiment of one minute's duration by the modified method is equal in accuracy to a two hours' experiment by the older method.

It is perhaps hardly necessary to refer to the great convenience of the modified method. For experiments in the open air sets of weighed tubes can be carried even for long distances in a box with places arranged for the tubes. We have found that no variation in weight is caused by the shaking, \&c. (see p. 317). No precautions beyond the very simple ones above mentioned are necessary in weighing; and with a good short-beam balance not more than three minutes are occupied in the whole of the manipulations connected with weighing. Once an absorption-apparatus is filled it is always ready for use, and may bo used every day for nearly a year, The fact that only a small quantity (usually about 6 litres) of air is needed for an analysis makes it possible to use an easily portable aspirator, such as that described above.

\section{Comparative Experiments with the Dry-and Wet-Bulb}

Psychrometer and the Apparatus described above. (Made at the Radcliffe Observatory, Oxford, by M. S. P.)

This series of experiments was undertaken with a view to test the degree of accuracy attainable by ordinary observations with the dry-and wet-bulb psychrometer ; and also with the object of obtaining independent evidence as to the accuracy of the different tables used in connexion with the psychrometer.

The chemical method has been recognized by Regnault and others as the standard with which to compare all other hygrometers. How important the first of these observers considered the method is shown by the following extracts from his Etudes sur l'Hygrométrie :-“. . . j'attachais un grand intérêt à rendre cette méthode éminemment pratique et facile à employer dans toutes les expériences hygrométriques." *

“La méthode chimique convient éminemment à la vérification des autres méthodes hygrométriques et à la détermination des constantes numériques que plusieurs d'entre elles exigent. J'en ai constamment fait usage, à ce point de vue, dans les recherches qui font l'objet de mon premier Mémoire." †

* Annales de Chimie et de Physique, xv. p. 152 (1845).

\& Ibid. xxxvii. p. 257 (1853). 
Although Regnault proved by experiment that the absorption of moisture was complete with the chemical method, yet he was prevented from bringing it into general use in hygrometry by two difficulties. These were the inaccuracy of the weighings, and the slow rate at which the air could be aspirated through the absorption-tubes * . All other observers who have used the chemical method have had to encounter these same difficulties. Even the mercury-joints and glassstoppered absorption-tubes used by Shaw have not removed the errors arising from these causes $\dagger$. Each determination made by these observers required about one hour, and generally longer. By what simple means these serious errors can be avoided has been shown above.

I must here express my hearty thanks to the Radcliffe Observer for giving me every facility to carry out these experiments. To his assistants Messrs. Wickham, Robinson, and Bellamy, my thanks are also due.

Two determinations were made each time and as nearly simultaneously as possible. Two aspirators similar to the one described above were used. Four pairs of absorption-tubes were employed each time :-pair 1 for determination A ; pair 2 for determination $B$; pair 3 as a test poir to show if any alteration in weight was caused by carrying the tubes from the Physiological Laboratory (where the tubes were weighed) to the Radcliffe Observatory and back ; whilst pair 4 was the counterpoise.

The tubes were carried in a small box with partitions of copper wire to prevent them from knocking against each other. The entrance tube, by which the air to be examined passed into the pair 1 (or 2), was fixed through a small perforation in a rubber partition covering a hole in the box, so that there was no possibility of air being taken from the inside of the box. The box and its tubes were placed about 1 foot below the wet and dry bulbs. That the air in this position might be fairly compared with that in the shed containing the psychrometer is shown by experiment 6 , in which pair 2 was placed in the shed close to the bulbs, and pair 1 in the usual place below.

In making a determination the following was the order of

* Ann. de Chim. et de Phys. xv. pp. 153 and 164. "La méthode chimique est trop embarassant, et elle exige une manipulation trop longue pour qu'on puisse l'employer' souvent dans les observations méteorologiques."

$\uparrow$ Shaw on Hygrometric Methods, Phil. Trans. 1888, A, pp. 83 and 84 . The variations in weight range from -.0009 to +.0023 gram. or still higher. 


\section{Messrs. Haldane and Pembrey on an Improved Method}

procedure. The wet and dry bulbs were first read off ; the absorption-tubes, pairs 1 and 2, were then connected by two long pieces of rubber tuling with their respective aspirators, and the air was drawn through them at as equal a rate as possible. The readings of the thermometers in the aspirators were now taken. When one known volume (2900 cub. cent.) had been drawn through, the aspirators were quickly reversed and started again until 5800 cub. cent. of the air had been taken for each determination. The thermometers in the aspirators were now again read; the readings of the wet and dry bulbs entered; the apparatus disconnected; and the absorption pairs 1 and 2 stoppered. Everything was easily done in twelve minutes, whilst the actual period of aspiration was only about half this time, as shown by the Table of experiments.

How closely the two determinations agree Table I. will show, and there is no doubt that could they have been strictly simultaneous the agreement would have been still closer. This is well shown by a comparison of the first five or six experiments with the last four, in which the determinations were almost exactly simultaneous.

To show that no practical error was introduced in the weighings by carrying the tubes about, the variation of the test pair 3 is given in Table $I$.

In calculating the results for the psychrometer in Table I. I have taken the mean of three readings-one at the beginning, one at the middle, and one at the end of each period of aspiration (i.e. one reading about every two minutes). These readings were obtained from a continuous photograpbic record of the wet and dry bulb taken at the Radcliffe Observatory. This record can be read off to two minutes and one tenth of a degree Fahrenheit, and its accuracy has been proved by years of use and comparison with eyereadings. The volume of air drawn through the tubes has always been corrected for temperature, aqueous vapour, and barometric height. In calculating the tension of aqueous vapour from the chemical determinations I have used the table given by Shaw in his paper on Hygrometric Methods*.

The mean of all these gravimetric determinations compared with the mean of the amounts of moisture ealculated from the psychrometric readings is 0551 to $0549 \mathrm{grm}$., or less than $+\frac{1}{2}$ per cent. Thus although the psychrometric result varied from the standard chemical method in the above series by +6 per cent. to -5 per cent., the mean difference is insensible.

* Phil. Trans. 1888, A, pp. 78, 79. 
of Determining Moisture and Carbonic Acid in Air. 317

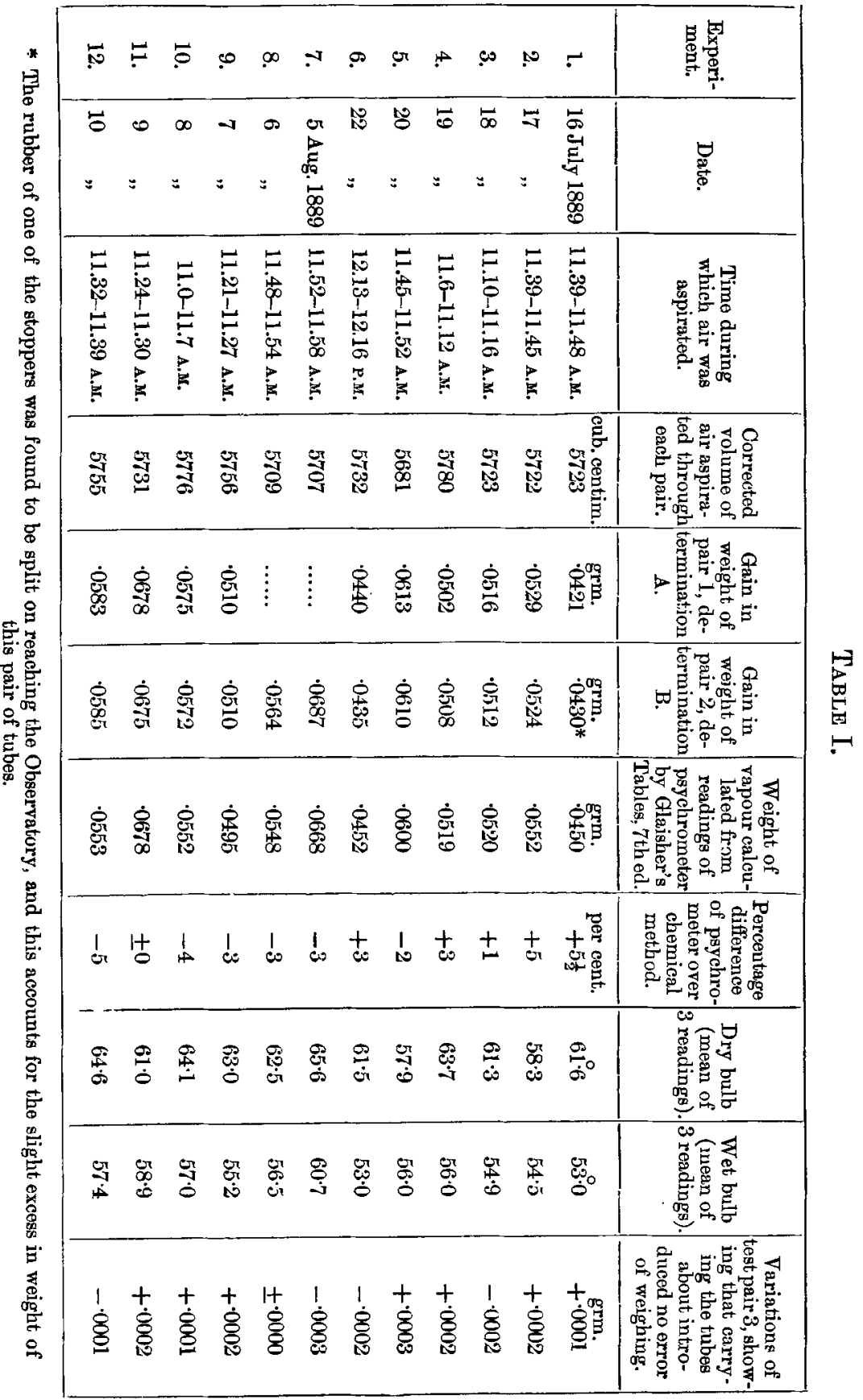




\section{Messrs. Haldane and Pembrey on an Improved Method}

During some experiments the psychrometer was very steady; at other times it showed considerable fluctuations. As examples may be given the readings of the wet and dry burlbs for the experiments 1 and 5 .

\begin{tabular}{|c|c|c||c|c|c|}
\hline Exp. 1. & Dry. & Wet. & Exp. 2. & Dry. & Wet. \\
\hline 11.39 A... ...... & 61.4 & 52.9 & 11.45 A.M. ..... & 57.9 & 55.9 \\
$11.43 \ldots \ldots \ldots \ldots$. & 61.8 & 53.1 & $11.49 \ldots \ldots \ldots \ldots$ & 57.9 & 56.0 \\
$11.47 \ldots \ldots \ldots \ldots$. & 61.6 & 52.9 & $11.52 \ldots \ldots \ldots \ldots$ & 58.0 & 56.0 \\
\hline
\end{tabular}

It is to be noticed that a difference of two or three tenths of a degree Fahrenheit in the reading alters greatly the percentage error.

I have also calculated out the tension given by the chemical method, and have compared it with the results calculated from the psychrometer by means of Glaisher's, Haeghen's, Guyot's, and Wild's Tables.

TABLE II.

\begin{tabular}{|c|c|c|c|c|c|}
\hline \multirow{2}{*}{ Experiment. } & \multirow{2}{*}{$\begin{array}{l}\text { Chemical } \\
\text { Method. }\end{array}$} & \multicolumn{4}{|c|}{ Psychrometer. } \\
\hline & & Glaisher. & Haeghens. & Guyot. & Wild. \\
\hline & $\operatorname{mm}$. & & & & \\
\hline $1 \quad \ldots \ldots \ldots \ldots$ & $\begin{array}{l}7 \cdot 43 \\
9 \cdot 15\end{array}$ & $\begin{array}{l}7 \cdot 76 \\
9 \cdot 55\end{array}$ & $\begin{array}{l}7 \cdot 37 \\
9 \cdot 54\end{array}$ & $7 \cdot 31$ & $\begin{array}{l}7 \cdot 4 \\
9 \cdot 5\end{array}$ \\
\hline $\begin{array}{l}2 \\
3 \\
3\end{array} \ldots \ldots \ldots \ldots \ldots$ & $\begin{array}{l}9 \cdot 15 \\
8.99\end{array}$ & $\begin{array}{l}9 \cdot 55 \\
9 \cdot 00\end{array}$ & $\begin{array}{l}9.54 \\
8.88\end{array}$ & $\begin{array}{l}9.51 \\
8.87\end{array}$ & $\begin{array}{l}9 \cdot 5 \\
8 \cdot 9\end{array}$ \\
\hline $4 \ldots \ldots \ldots$ & 8.78 & 8.99 & $8 \cdot 74$ & $8 \cdot 77$ & 8.8 \\
\hline $5 \quad \ldots \ldots \ldots \ldots$ & $10 \cdot 70$ & $10 \cdot 72$ & $10 \cdot 75$ & 1076 & 107 \\
\hline & $7 \cdot 63$ & $7 \cdot 78$ & $7 \cdot 39$ & $7 \cdot 34$ & $7 \cdot 4$ \\
\hline $7 \ldots \ldots \ldots$ & $12 \cdot 15$ & $11 \cdot 72$ & $11 \cdot 78$ & $11 \cdot 80$ & $11 \cdot \overline{7}$ \\
\hline $8 \ldots \ldots \ldots \ldots$ & 9.91 & $9 \cdot 65$ & $9 \cdot 57$ & $9 \cdot 57$ & $9 \cdot 6$ \\
\hline 9 ....................... & 8.90 & $8 \cdot 68$ & $8 \cdot 45$ & $8 \cdot 43$ & $8 \cdot 5$ \\
\hline $10 \ldots \ldots \ldots \ldots$ & $10 \cdot 00$ & 9.52 & $9 \cdot 38$ & $9 \cdot 41$ & $9 \cdot 4$ \\
\hline $11 \ldots \ldots \ldots$ & 11.82 & $11 \cdot 84$ & 11.93 & 11.93 & 11.9 \\
\hline $12 \ldots \ldots \ldots \ldots$ & $10 \cdot 23$ & 967 & $9 \cdot 55$ & 9.57 & $9 \cdot 5$ \\
\hline Mean ...... & $9 \cdot 64$ & 9.57 & $9 \cdot 44$ & $9 \cdot 44$ & $9 \cdot 44$ \\
\hline
\end{tabular}

This Table shows that Glaisher's Tables are the most correct as far as my experiments go. These tables were prepared by Glaisher from the Greenwich factors and Regnault's Table of pressures, and are in general use in England. Haeghen's Tables, which are used in France and Italy, Guyot's, which are almost identical with the last and are employed in America, and Wild's, which have been adopted 
by Germany and Russia, are all reduced from Regnault's table of pressure and psychrometric formulæ.

In conclusion may be given the results obtained by other observers, who have compared the psychrometer with the chemical method. Regnault *, who made 106 experiments under very varied conditions, obtained for the mean percentage error 2, whilst the extreme percentage error varied from +12 to -10 . In order to calculate out the results from the readings of the dry and wet bulbs, Regnault determined the values for the constant $A$ in each series of experiments. Each experiment lasted about one hour ; the readings of the psychrometer were taken every five minutes, and from the mean of these the result was calculated. M. Izarn made 34 comparative experiments in the Pyrenees. These are given by Regnault in his paper $\uparrow$. The extreme percentage differences are +2 and -3 in the first series, and +10 and -10 in the second.

Shaw made comparisons in a room with a current of air passing over the instruments; he does not, however, consider them satisfactory. The tensions calculated from the psychrometer by Glaisher's Tables were generally higher than those given by the chemical determination, the variations ranging from +30 per cent. to -7 per cent. $\ddagger$.

\section{The Determination of Carbonic Acid.}

The method adopted by us for carbonic acid is similar in principle to that for moisture. The absorption-apparatus (see fig. 1) consists of a pair of test-tubes arranged in the same way as was described above (p. 307) §. The first tube is filled with soda-lime, and the second with sulphuric acid and pumice. The air must arrive perfectly dry at the absorptiontube for carbonic acid, and is therefore passed through the previously described absorption-apparatus for moisture. The connecting piece of rubber tubing should be as short as possible.

The soda-lime we bave hitherto used was made by heating together in a copper vessel 1200 grms. of caustic soda in strong solution, and the product obtained by slaking to a fine powder 1000 grms. of quicklime. The heating was continued until the soda-lime became capable of being broken up into fragments in a mortar. The fragments were rapidly sifted

* Annales de Chimie, xxxvii. pp. 264-285 (1835).

$\uparrow$ Ibid. pp. 275-272.

† Phil. Trans. 1888, A, p. 111.

$\S$ The same counterpoise is used for the carbonic acid as for the moisture determinations. 
in a wire sieve with about 12 meshes to the inch, and the product freed from powder by a finer sieve. We have also tried commercial soda-lime similarly sifted, and found that it absorbed $\mathrm{CO}_{2}$ well (see p. 321). As a precaution against blocking of the tubing by soda-lime during the process of fitting, a small plug may be placed in the end of the tube and afterwards be withdrawn by a string attached to it. The figure shows that a piece of cotton-wool is placed between the surface of the soda-lime and the cork ; the object of this is to prevent soda-lime being carried over in the form of powder by a rapid air-current.

The method was tested in the same way as that for moisture. As regards accidental errors of weighing, what was said above with regard to the apparatus for moisture applies equally to that for carbonic acid. With respect to the efficiency of the absorption, we may first quote the results of two experiments in which the soda-lime and sulphuric-acid tubes were weighed separately. In the subsequent experiments the double absorption-tubes just described were employed.

\section{Experiment No. 1.}

The tubes were arranged in the following order;-1 and 2 , sulphuric acid; 3 , soda-lime; 4, sulphuric acid; 5 , sodalime ; 6, sulphuric acid. 90 litres aspirated at a rate of 1 litre per minute.

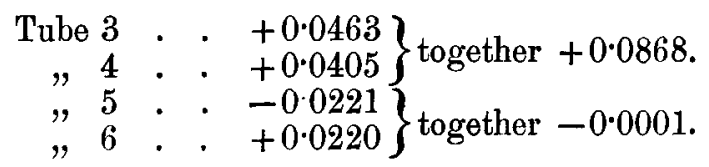

Experiment No. 2.

Repetition of No. 1;90 litres aspirated at a rate of 1 litre per minute.

$$
\left.\begin{array}{rlll}
\text { Tube } 3 & : & \cdot & +0.0360 \\
\Rightarrow 4 & \cdot & \cdot & +0.0838
\end{array}\right\}+0.1198 .
$$

\section{Experiment No. 3.}

90 litres aspirated at a rate of 1 litre per minute.

$$
\begin{aligned}
& \text { Soda-lime, pair } 1 . \quad+0.1615 \text {. } \\
& \text { " } 2 . \quad+0.0001 \text {. }
\end{aligned}
$$


Experiment No. 4.

50 litres aspirated at a rate of $1 \frac{3}{4}$ litres per minute.

Soda-lime, pair 1 . . +0.0558.

"

, $2 .+0.0001$.

Experiment No. 5.

21 litres aspirated at a rate of 2 litres per minute.

Soda-lime, pair $1 . \quad+0.0339$.

$, \quad, 2 . .+0.0003$.

Experiment No. 6.

In this experiment commercial soda-lime (Hopkins and Williams) was used.

The soda-lime contained much more water than ours, as shown by its appearance and the amount of water it gave up to dry air. 50 litres aspirated at a rate of 1 litre per minute. Soda-lime, tube 1 . . . -0.0552$\}$

Sulphuric acid, tube $3 .+0.0923\}$ together +0.0371 . Soda-lime, pair 2 . . . $+0 \cdot 0000$.

\section{Experiment No. 7.}

The same soda-lime tube was used as in the previous experiment. 50 litres aspirated at a rate of 2 litres per minute.

Soda-lime, tube $1 . . \quad \cdot-0.0478\}$

Sulphuric acid, tube $3: \quad+0.0893\}+0.0415$.

Soda-lime, pair 2. . . + +0.0013.

A rate of 1 litre a minute is thus well within the limit of safety, so that even should the aspiration be temporarily faster, there is no risk of appreciable non-absorption of either moisture or carbonic acid.

The number of analyses possible without refilling the carbonic-acid tubes was determined in the same way as for the moisture-tubes, $i . e$. by passing air through two pairs at a rate of 1 litre per minute, and weighing the second tube at intervals.

\section{Experiment No. 8.}

120 litres of air had already been passed throngh pair No. 1 .

Litres of air aspirated through pair 1.
Variation in weight

of pair 2.

$+0.0003$

$-0.0001$

$+0.0010$

$+0.0049$ 


\section{Messrs. Haldane and Pembrey on an Improved Method}

At the end of the experiment pair No. 1 had absorbed 0.4371 gram of carbonic acid from 688 litres of air. We concluded that 500 litres of air might be safely passed through an absorption-apparatus. Other experiments confirmed this conclusion.

The mean error of the method for carbonic acid was estimated in the same way as with the method for moisture (p. 311).

\section{Total $\mathrm{CO}_{2}$ absorbed by tubes No. $1 . \quad . \quad 0.6464$}

Sum of variations of tubes No. $2 \quad . \quad+0.000 \tau$

The mean error was thus about $0 \cdot 1$ per cent.

The method is thus exceedingly accurate, in this respect far exceeding, as will be shown below, the other methods in common use.

For free air about 20 litres or more, according to the accuracy aimed at, will be required for an analysis. For vitiated air, such as that of schools, 3 to 6 litres will suffice.

The result of a pair of simultareous experiments made out of doors may be quoted here. 18 litres of air were aspirated in each case.

$$
\text { No. } 1 .
$$

$$
\begin{aligned}
& \text { Sulphuric acid pair . . . . }+0.0820 \\
& \text { Soda-lime pair . . . . . +0.0109 }
\end{aligned}
$$

No. 2.

Sulphuric acid pair . . . . $+0 \cdot 0819$

Soda-lime pair . . . . . + +0.0109

The methods just described have now been in use in this laboratory for nearly a year. They have also been used for some months in experiments out of doors at the Radcliffe Observatory here, and in the country in Scotland. So far as our own experience of them goes they have successfully stood the test of every-day use.

We may now review shortly the literature bearing on the determination of carbonic acid in air, and record some experiments which we made with a view to testing the two methods at present in common use in this country.

The first attempt at a quantitative analysis of the carbonic acid in free air seems to have been made by A. von Humboldt *. His numerous results were, curiously enough, entirely illusory, since he concluded that about 100 vols. per 10,000 of air were usually present. In 1802 Dalton $\dagger$, who

* Gilhert's Annalen, iii. p. 79 (1800).

† Manchester Lit. Phil. Soc. Mem. 1802. 
made the first approximately correct analyses, reduced the amount to 6.8 vols. ; and De Saussure * had, about 1830 , brought it down to a mean of about $4 \cdot 1$ vols. for country air. This result was confirmed by Watson (1834) and Boussingault (1844), who used other methods, and for long was supposed to be nearly correct. Still more recent researches, particularly those of Angus Smith $\dagger$ in Scotland, Schultze in Germany, Reiset $\ddagger$ in France, and Müntz and Aubin in France and other countries, have gradually reduced it by about a third more.

There still, however, exists a considerable amount of apparent contradiction on the subject. Not only are the mean results of different observers different, but there is perhaps still greater want of agreement as regards the limits within which the proportion of $\mathrm{CO}_{2}$ varies. That the uncertainty and want of agreement which prevails is probably due to the imperfection of some, at least, of the methods employed will, we believe, be evident from the experiments to be recorded below. The subject of the variations in the carbonic acid of free air will, however, be taken up in a future paper.

The methods at present in use for the determination of carbonic acid in air are, practically speaking, of two kinds : (1) those in which the $\mathrm{CO}_{2}$ is absorbed by standardized baryta-water, which is afterwards retitrated; (2) those in which it is absorbed by potash, and afterwards liberated by acid and measured in the form of gas $\|$.

The method best known in this country is that of Pettenkofer 9 , or, more correctly, of Dalton** and Pettenkofer. In this method a large bottle is pumped full of the air to be examined. A measured quantity of standardized baryta-

* Annales de Chimie, xliv. p. 5 .

+ 'Air and Rain,' p. 59.

$\ddagger$ Annales de Chimie, xxvi. p. 145 (1882).

\$ Ibid. xxvi. p. 222 (1882), xxx. p. 238 (1883).

II A valuable critical account of the methods hitherto used is given by Blochmann, Liebig's Annalen, cexxx vii. p. 39 (1887).

If Abhandl. der techn. Commission der Bayer. Akad, ii. p. 3 (1858); and Liebig's Annalen, Suppl. ii. p. 23 (1861).

** The form finally given to Dalton's method is described in a paper by Watson, communicated by Dalton to the British Association (Brit. Assoc. Report, 1834, p. 583). Watson used a large bottle, which he filled with air with the help of a pair of bellows. He then added limewater, and after shaking allowed the bottle to stand for several days, at the end of which time he filtered off the carbonate, and titrated with dilute sulphuric acid. Pettenkofer independently invented a similar but improved method. He introduced baryta-water as an absorbent, and turmeric as an indicator, and avoided the filtration. Watson's mean result for country air ( $4 \cdot 1$ vols.) was about the same as that obtained by the earlier observers who used Pettenkofer's method. 
water is then added, and the bottle closed and shaken, so as to bring the air in contact with the baryta. After a certain time the baryta solution is again titrated, and the $\mathrm{CO}_{2}$ estimated from the loss of alkalinity of the baryta-water.

For certain cases where the bottle method is not suitable, Pettenkofer recommends that a measured quantity of air should be allowed to bubble through a long tube placed nearly horizontally, and containing a known quantity of baryta-water. The $\mathrm{CO}_{2}$ absorbed by the baryta is estimated in the same way as in the first method. Pettenkofer found that about 5 litres per hour is the maximum rate at which the air can be passed.

Reiset* has recently devised an apparatus which allows large quantities of air to be aspirated through baryta-water. With this apparatus he was able to aspirate as much as 100 litres through 300 cub. cent. of baryta-water in an hour. The absorption of $\mathrm{CO}_{2}$ appears to have been complete. Each experiment lasted several hours. He used an enormous aspirator, mounted on a cart, and capable of holding 600 litres of water.

The method in which the $\mathrm{CO}_{2}$ is absorbed by potash, and afterwards liberated by acid and measured in the form of gas, was in its first form used by Mangon and Tissandier $\dagger$ for balloon experiments. A rough form of this method has been for some years in use at the Paris Observatory. The method has been brought to great perfection by Müntz and Aubin $\ddagger$, whose test-experiments show that an accuracy to about 2 per cent. of the $\mathrm{CO}_{2}$ estimated may be attained when 200 litres of air are used. For absorbing the $\mathrm{CO}_{2}$ they use tubes about a metre long, filled with pumice soaked in caustic-potash solution free from $\mathrm{CO}_{2}$. After the experiment the $\mathrm{CO}_{2}$ is liberated by the addition of acid, and collected over mercury with the help of a mercury-pump. The apparatus and manipulations required are very complicated, so that the method is not likely to come into general use. Müntz and Aubin confirmed and extended Reiset's conclusions, and found that the mean proportion of $\mathrm{CO}_{2}$ in country air is about $2 \cdot 85$ vols. per 10,000 , and that it very seldom rises above $3 \cdot 1$ or falls below $2 \cdot 6$. The experiments of these three observers are certainly the most reliable of any hitherto published.

Gravimetric methods of determining $\mathrm{CO}_{2}$ in air were at one time much employed, although they have been practically disused since the introduction of Pettenkofer's method. In

* Loc. cit. p. 164.

$\dagger$ Comptes Rendus, lxxx. p. 976 (1875).

$\ddagger$ Annales de Chemie, xxvi. p. 222 (1882). 
1832 Brunner* described a gravimetric method. He aspirated the air at a rate of about 30 litres an hour through two absorption-tubes. The first contained asbestos soaked in sulphuric acid ; and the second, which was 3 feet long, was filled in the first two thirds with moist slaked lime, and in the last third with sulphuric acid and asbestos. He tested the completeness of the absorption of the $\mathrm{CO}_{2}$ by allowing the air to bubble afterwards through baryta-solution, which remained clear. The increase in weight of the first tube gave the moisture, that of the second the $\mathrm{CO}_{2}$ in the air aspirated. $\mathrm{He}$ says he always obtained for free air results lying between De Saussure's maximum and minimum $(3 \cdot 7$ and $6 \cdot 2$ vols. per 10,000).

Brunner's method, with various modifications, has been used by a number of subsequent observers. The most reliable results were those of Boussingault, who obtained with large volumes of air a mean of $4 \cdot 0$ vols. per 10,000 for Paris air $\dagger$ The results of several other observers were certainly quite unreliable.

In 1856 Hlasiwetz $\ddagger$ published a paper in which he criticised adversely the gravimetric method. He made a number of analyses by different forms of this method, and found that not only did analyses simultaneously made often entirely disagree, but that in eleven out of fifty-six analyses the absorptionapparatus for $\mathrm{CO}_{2}$ actually lost in weight. These results he set down partly to unavoidable "errors of weighing," and partly to the fact that $\mathrm{CO}_{2}$ is absorbed by sulphuric acid. Hlasiwetz's criticisms appear to have been generally accepted as conclusive; and since the publication of his paper the gravimetric method has been almost entirely given up, though still described in some text-books.

Soda-lime has for long been in use as an absorbent for $\mathrm{CO}_{2}$ in combustions. It was first used by Mulder $\$$ in 1859, and Fresenius $\|$ has shown its superiority to potash-solution \&c. Its successful use by one of us in a new form of animal-

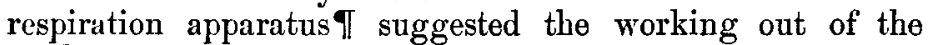
method described above.

We have made experiments with a view to testing by our

* Annalen der Physique, xxiv. p. 570.

+ Annales de Chimie, x. p. 456 (1844).

$\ddagger$ Sitzbes. der Wiener Akad. (Math.-nat. Kl.) xx. p. 189.

$\$$ Zeitschr. für Anal. Chem. i. p, 8.

॥ Ibid. v. p. 89.

I A full account of this apparatus will shortly be published. A preliminary paper on the subject was published in the 'Proceedings of the Oxford 'University Junior Scientific Club' for October 'Term, 1888.

Phil. Mag. S. 5. Vol. 29. No. 179. April 1890. 2 C 


\section{Messrs. Haldane and Pembrey on an Improved Method}

gravimetrie method the bottle method and also the tube method of Pettenkofer.

The bottle method is in general use in this country and in Germany, and is in many ways exceedingly convenient. There are reasons, however, for suspecting that even in experienced hands it may give seriously inaccurate results for freo air. Thus although the French experiments referred to above, and a series at present in progress by our own method, show that the real proportion of carbonic acid is, as a rule, under 3 vols. per 10,000 , and fluctuates within pretty narrow limits; yet with the Pettenkofer method a very different average proportion is often obtained, and the apparent limits of fluctuation are, as a rule, much greater.

We may quote a few recent instances. Marcet and Landriset * found at two country stations near Geneva $2 \cdot 7$ to $4 \cdot 9$ vols. per 10,000, mean $3 \cdot 76$ vols. Carnelley, Haldane, and Andersont, who titrated with the barium carbonate in suspension, found in the outskirts of Dundee and Perth 1.7 to 3.5 vols., mean $2 \cdot 9$. Feldt $\ddagger$ who also titrated with the carbonate in suspension, found in Dorpat 1.85 to $3 \cdot 65$, mean $2 \cdot 66$ vols. The results of pairs of simultaneous analyses made by him sometimes disagreed by as much as 30 per cent. Üffelmann $\S$, in Rostock, found a mean of 3.5 vols., whereas Schultze at the same place had previously obtained an average of 2.9 vols. Yet these three observers seem to have used very nearly the same modification of Pettenkofer's method. All titrated straight into the absorption-bottle, the carbonate being still in suspension.

In the following comparative experiments, which were nearly all made within doors, every care was taken to obtain the air from the same place in the room, and at the same time in each pair of determinations. The bottles were pumped full of air when the aspiration through the tubes was half over.

For the Pettenkofer analyses we used two large bottles of 10,680 and 10,800 cub. cent. capacity, with ground-glass stoppers $\|$. Before each analysis these bottles were washed, first with tap-water, and then with distilled water, and dried. They were not washed with acid. The air was pumped into the bottle with a bellows, 100 cub. cent of baryta-water

* R. Meteorol. Soc. Journ. xiii. p. 167 (1887).

† Phil. Trans., B, 1887, pp. 66, 68.

† Der Kohlensaïregehalt der Luft zu Dorpat, 1887.

$\$$ Archiv für Hygiene, viii. p. 252 (1888).

II India-rubber caps are objected to by Blochmann, Liebig's Annalen, cexxxvii. p. 39 (1887). 
of Determining Moisture and Carbonic Acid in Air. $\quad 327$

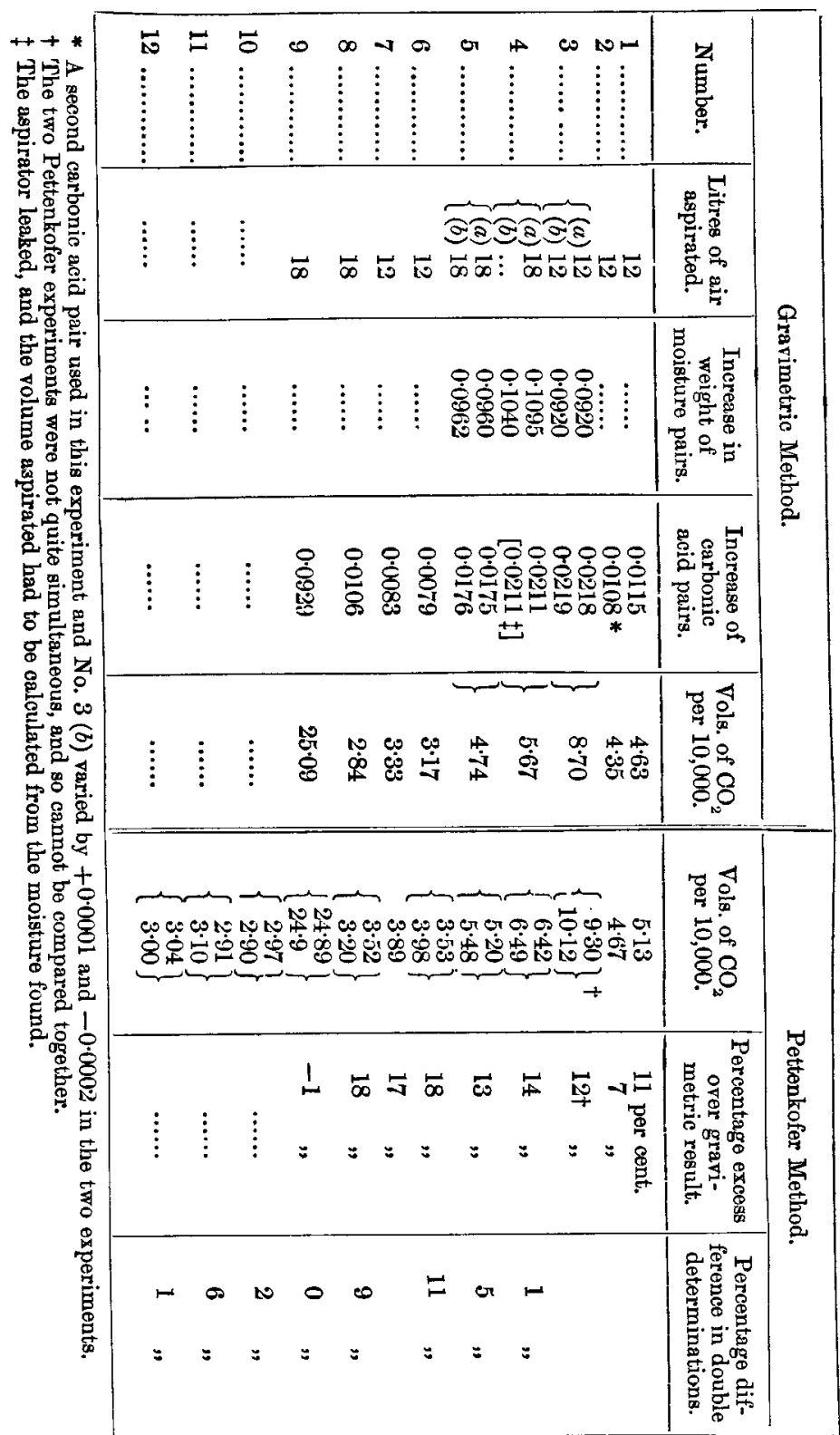

$2 \mathrm{C} 2$ 


\section{Messrs. Haldane and Pembrey on an Improved Method}

added, and the bottle allowed to stand, with occasional shaking, for at least two hours. The baryta-water was then removed to a clean and dry cylinder, corked up, and titrated after the carbonate had subsided. We used as a rule either 30 or $40 \mathrm{cub}$. cent. of the baryta-water in the titration, which was performed twice with the ordinary oxalic-acid solution ( 1 cub. cent. $=\cdot 001$ gram. of $\left.{ }^{\ddagger} \mathrm{CO}_{2}\right)$, rosolic acid or phenolphthalëine being used as an indicator. The end reaction was always sharply defined, and the two results never differed by more than $0 \cdot 1$ cub. cent.

The above Table shows that with air containing a small proportion of carbonic acid the Pettenkofer method gave results from 8 to 27 per cent. higher than those given by the gravimetric method. As the proportion of carbonic acid rises (Experiment No. 9), the difference tends to disappear. Judging from these experiments the result for outside air would usually be about 15 or 20 per cent. too high by the bottle method.

It will be seen that the pairs of determinations made with the gravimetric method agree with the utmost closeness, both for moisture and carbonic acid.

We have not succeeded in tracing satisfactorily the reasons for the inaccuracy of our results with the bottle method. Although these results were all too high, other observers have, as before mentioned, obtained results which were often almost certainly too low.

We at first thought that the error might be due to contact of the baryta-water with air during the manipulations involved. In the first four or five analyses we had simply followed Pettenkofer's plan of pouring the baryta-water out of the large bottle through a funnel into the narrow vessel used for allowing the carbonate to settle. To avoid the exposure to air implied in this operation, we in the later experiments removed the baryta-water with a pipette provided with a piece of rubber tubing for applying suction. As the pipette was washed first with some of the baryta, it was free from carbonic acid. Our results, however, were little, if at all, better.

Another possible source of error is the state of the glass walls of the bottle. Pflüger ${ }^{*}$ found in making the titrations for Kjeldahl nitrogen determinations that it was impossible to titrate accurately in flasks which had previously been washed with acid. The glass seemed to retain some of the acid in spite of washing, and give it up again slowly during the analysis. We made several experiments with a bottle

* Pflüger's Archiv, xxxvi. p. 105. 
which had been washed with mineral acid and then allowed to stand full of water for a day; but the result was not always higher with this bottle.

In the bottle experiment No. $6(b)$, which gives the worst result of all, a smaller bottle of about 3 litres capacity was used. In Parkes's 'Hygiene,' 7th edition (1887), p. 711, it is stated that the results by the bottle method vary with the different sizes of bottle. It seems likely that as a rule the results will be less correct the smaller the bottle used.

When the results are too low this is as likely to be due to insufficient time being given for the carbonate to settle out as to incomplete absorption. If the attempt is made to titrate too soon, the result may be much too low, and the end reaction will be very indefinite.

It is no conclusive test of the correctness of the bottle method that two simultaneous experiments give the same result. If the conditions are kept the same in the two experiments, the results must be the same whether right or wrong. In the above experiments we usually varied the conditions somewhat as regards details supposed to be unessential. In experiments where the conditions were carefully kept the same, results were practically identical. There are doubtless several sources of error, which may neutralize one another more or less. The subject is complicated by the fact that nearly every observer introduces, often unconsciously, some new modification of the method.

We have come to the conclusion from the above experiments that the bottle method in its ordinary form is of very limited use for experiments on free air, though usually quite accurate enough in the case of vitiated air.

Biochmann * has recently described a modification of the bottle method which enables him both to avoid all possibility of contact of the baryta-water with air, and at the same time to titrate with perfectly clear baryta-water. For want of the special apparatus required we have not yet tested this method.

We used Pettenkofer's tube method in some of our earlier experiments for the purpose of testing the efficiency of the absorption of carbonic acid by soda-lime. Before we had overcome the difficulty caused by errors of weighing, we believed that any carbonic acid which had escaped absorption by the soda-lime would be indicated best by Pettenkofer tuhes filled with baryta-water. Accordingly in different experiments the air was passed through one or more Pettenkofer tubes after it had passed through the soda-lime. We always found that the baryta-water had lost distinctly in alka* Liebig's Annalen, cexxxvii. p. 72 (1887). 
linity after the experiment. There were, however, several reasons for doubting whether this was really due to escape of carbonic acid through the soda-lime. We therefore made the following experiment.

A current of air, after passing through sulphuric acid and soda-lime absorption-tubes, was divided by means of T-tubes, and passed through six Pettenkofer tubes placed beside one another on a board. The loss of moisture from the barytawater in these tabes was determined by means of a sulphuric acid absorption-tube placed in the air-current beyond them. The moisture which had collected in the tubing connecting the Pettenkofer tubes and this absorption-flask was washed into the flask by means of a current of dried air. 60 litres of air were aspirated in one hour.

In addition to the six Pettenkofer tubes through which the air was passed six others were taken, and charged with the same quantity of baryta solution in exactly the same way as the first set. These dummy tubes were kept stoppered during the experiment.

All the tubes were washed before the experiment, first with dilute nitric acid, then three times with tap-water, and finally with distilled water. At the end of the experiment the baryta from each set of tubes was poured into a small flask, and at once corked and allowed to stand for a time. During these manipulations every precaution was taken to avoid unnecessary contact of the baryta-water with air. The results are given in full.

$$
\begin{array}{ll}
\text { Sulphuric acid, pair 1. } & +0.4183 . \\
\text { Soda-lime, pair 1. } & +0.0554 .
\end{array}
$$

Baryta-waterfrom $\int$ (1) 40 c. c. $=39 \cdot 9$ c. c. standard oxalic. first set of Petten-

kofer tubes.

$$
\text { (2) }, \quad=39 \cdot 9
$$

Loss of water from same tubes by evaporation 0.8408 gram.

Baryta-water from $($ (1) 40 c.c. $=39 \cdot 65$ c. c. standard oxalic. dummy set of Pettenkofer tubes.

$$
(2), \quad=\frac{39 \cdot 75}{39 \cdot 7} " \quad, \quad,
$$

Standard baryta-water 40 c. c. $=40 \cdot 25$ standard oxalic.

Therefore

loss of alkalinity in 1 st set $=6 \times 0 \cdot 35=2 \cdot 1$ c. c. standard oxalic and loss by evaporation $=$

loss of alkalinity in 2nd set $=6 \times 0.55=\frac{0.84}{2 \cdot 94} \quad " \quad " \quad "$, 
Structure of the Line-Spectra of the Chemical Elements. 331

The dummy tubes had thus actually lost slightly more in alkalinity than the tubes through which the air had passed. This loss is about equal to what would be caused by the absorption of the carbonic acid contained in 6 litres of pure air.

It follows from this and the other experiments just referred to that the error with the tube method may be considerable, at least in experiments of not more than two or three hours' duration.

XXXIV. On the Structure of the Line-Spectra of the Chemical Elements. By J. R. RYDberg, Ph.D., Docent at the University of Lund*.

\section{(Preliminary Notice.)}

THE researches, the most important results of which are given in the following pages, will be published with full details in the Svenska Vetensk.-Akad. Handlingar Stockholm. They have extended hitherto only to the elements which belong to the groups I., II., III. of the periodical system ; there is, however, no reason to doubt but that the laws I have found can be applied in the same way to all elements.

In my calculations I have made use of the wave-numbers ( $n$ ), instead of the wave-lengtbs $(\lambda) ; n=10^{8} \cdot \lambda^{-1}$, if $\lambda$ be expressed in Angström's units. As will be seen, these numbers will determine the number of waves on 1 centim. in air $(760$ millim., $16^{\circ}$ C. according to Angström), and are proportional, within the limits of the errors of observation, to the numbers of vibrations.

1. The "long" lines of the spectra form doublets or triplets, in which the difference ( $v$ ) of wave-numbers of their corresponding components is a constant for each element.

This law, found independently by the author, has already been announced by $\mathrm{Mr}$. Hartley for $\mathrm{Mg}, \mathrm{Zn}, \mathrm{Cd}$. The values of the constant differences $(v)$ vary from $v=3 \cdot 1$ in the spectrum of Be to $\nu=7784.2$ in the spectrum of Tl. In each group of elements the value of $v$ increases in a somewhat quicker proportion than the square of the atomic weight. For instance :-

* Communicated by the Author. 